\title{
Transesophageal echocardiographic guidance for percutaneous closure of aortic pseudoaneurysm using a type II Amplatzer vascular plug -a case report-
}

\author{
Hyo Jin Kim ${ }^{1}$, Sangmin Maria Lee ${ }^{1}$, Kiick Sung ${ }^{2}$, I-Seok Kang ${ }^{3}$, \\ Jong-Hwan Lee ${ }^{1}$, Jeong Jin Min ${ }^{1}$, Eunhee Kim ${ }^{1}$, Jiyeon Park ${ }^{1}$, and \\ Jin Hyoung Park ${ }^{1}$ \\ Departments of ${ }^{1}$ Anesthesiology and Pain Medicine, ${ }^{2}$ Thoracic and Cardiovascular Surgery, ${ }^{3}$ Pediatrics, \\ Samsung Medical Center, Sungkyunkwan University School of Medicine, Seoul, Korea
}

Aortic pseudoaneurysm after cardiac surgery is a rare entity, but it is potentially fatal due to its clinical course along with higher morbidity and mortality rates. Instead of open surgical repair, percutaneous procedures have been introduced as other options for managing an aortic pseudoaneurysm. In this case report, we describe transesophageal echocardiography guidance for successful percutaneous closure of an aortic pseudoaneurysm located in the left ventricular outflow tract by using a type II Amplatzer vascular plug in a patient in whom open surgical repair was not recommended.

Key Words: Bipolar radiofrequency, Neoplasm metastasis, Spinal cord compression, Spine, Vertebroplasty.

Corresponding author: Jong-Hwan Lee, M.D., Ph.D.

Department of Anesthesiology and Pain Medicine, Samsung Medical Center, Sungkyunkwan University School of Medicine, 81, Irwon-ro, Gangnam-gu, Seoul 06351, Korea

Tel: 82-2-3410-1928, Fax: 82-2-3410-0361

E-mail: jonghwanlee75@gmail.com

ORCID: http://orcid.org/0000-0001-8249-5550

Hyo Jin Kim is now with the Department of Anesthesiology and Pain Medicine, Seoul Paik Hospital, Inje University School of Medicine, Seoul, Korea.

This article includes supplemental video clips.

Received: February 17, 2015.

Revised: 1st, May 13, 2015; 2nd, August 13, 2015; 3rd, October 2, 2015; 4th, October 29, 2015.

Accepted: November 2, 2015.

Korean J Anesthesiol 2016 August 69(4): 400-405 http://dx.doi.org/10.4097/kjae.2016.69.4.400
Aortic pseudoaneurysm (PsA) after cardiac surgery is a rare entity, but it is prone to rupture, thrombosis, distal embolization and fistula formation $[1,2]$. These fatal characteristics of PsA make surgical treatment necessary. Despite the relatively improved outcomes, open surgical treatment is challenging and the morbidity and mortality rates of PsA are still high $[3,4]$. Furthermore, surgical treatment of PsA located in the aorta or the left ventricular outflow tract (LVOT) can cause serious complications, such as aorto-mitral valve disruption or coronary compromise [5-7].

Recently, percutaneous approach for closure of PsA has been preferred due to its less invasiveness and it has the potential to become an alternative to surgical treatment especially in patients in whom surgical treatment is expected to carry a higher risk. However, due to the anatomical and procedural complexity, meticulous guidance of multi-modality imaging should be provided during percutaneous closure of PsA located in the aortic root or the LVOT.

In this report, the authors describe transesophageal echocar-

(c) This is an open-access article distributed under the terms of the Creative Commons Attribution Non-Commercial License (http://creativecommons.org/ licenses/by-nc/4.0/), which permits unrestricted non-commercial use, distribution, and reproduction in any medium, provided the original work is properly cited. 
diography (TEE) guidance, which was performed in addition to fluoroscopic guidance, for facilitating successful percutaneous closure of PsA located in the LVOT by using a vascular plug.

\section{Case Report}

A 17-year-old male with Marfan syndrome was admitted for the repair of a LVOT PsA which was detected on a routine postoperative follow-up imaging study. He underwent total 9 cardiac surgeries including Bentall operation, total arch and descending thoracic aorta replacement with tricuspid and mitral annuloplasty. Among these surgeries, two were performed for treating graft infection with abscess formation and the other surgeries were performed for repair of aortic dissection. The serial chest computed tomography (CT) scans showed that the diameter of PsA was increased from $30 \mathrm{~mm}$ to $33 \mathrm{~mm}$ in a year and that it was located just below the left main coronary arterial opening (Fig. 1). Transthoracic echocardiography (TTE) showed communicating flow across the PsA. Through the preoperative meeting among pediatric cardiologists, cardiac surgeons, and anesthesiologists, percutaneous intervention was chosen for the treatment of this patient because surgical repair was expected to carry a very high risk.

On arrival at the hybrid operating room, anesthesia was induced using thiopental rocuronium bromide. Anesthesia was maintained with desflurane in a mixture of oxygen and medical air. After anesthesia induction and orotracheal intubation was performed, a TEE (iE33 xMATRIX Echocardiography system, Philips, NV, USA) probe was placed and continuous TEE moni-

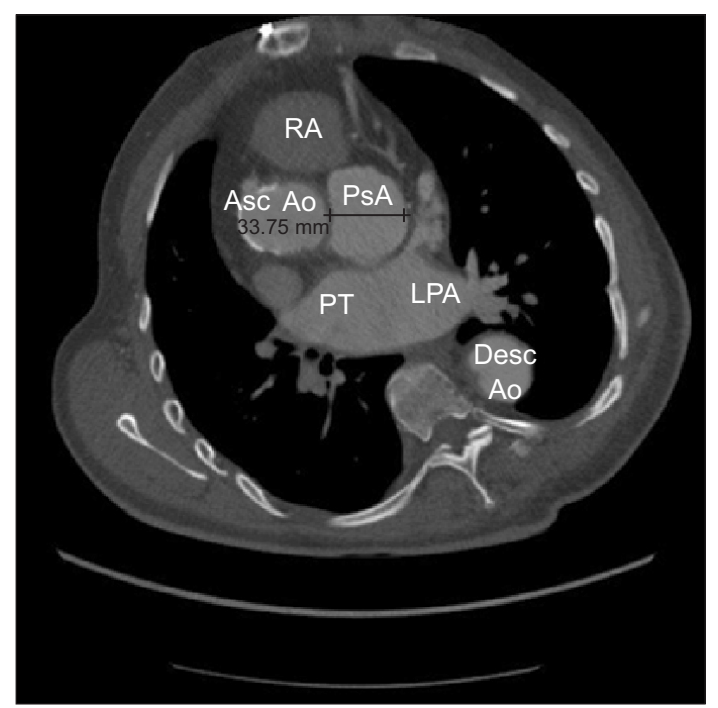

Fig. 1. Axial contrast-enhanced chest computed tomography image. The diameter of the aortic pseudoaneurysm (PsA) was $33.75 \mathrm{~mm}$ as indicated by the green line. RA: right atrium, Asc Ao: ascending aorta, PsA: pseudoaneurysm, PT: pulmonary trunk, LPA: left pulmonary artery, Desc Ao: descending aorta. toring was performed during the whole procedure by well-experienced cardiac anesthesiologists. Pre-procedural intraoperative TEE examination showed good left ventricular contractility with
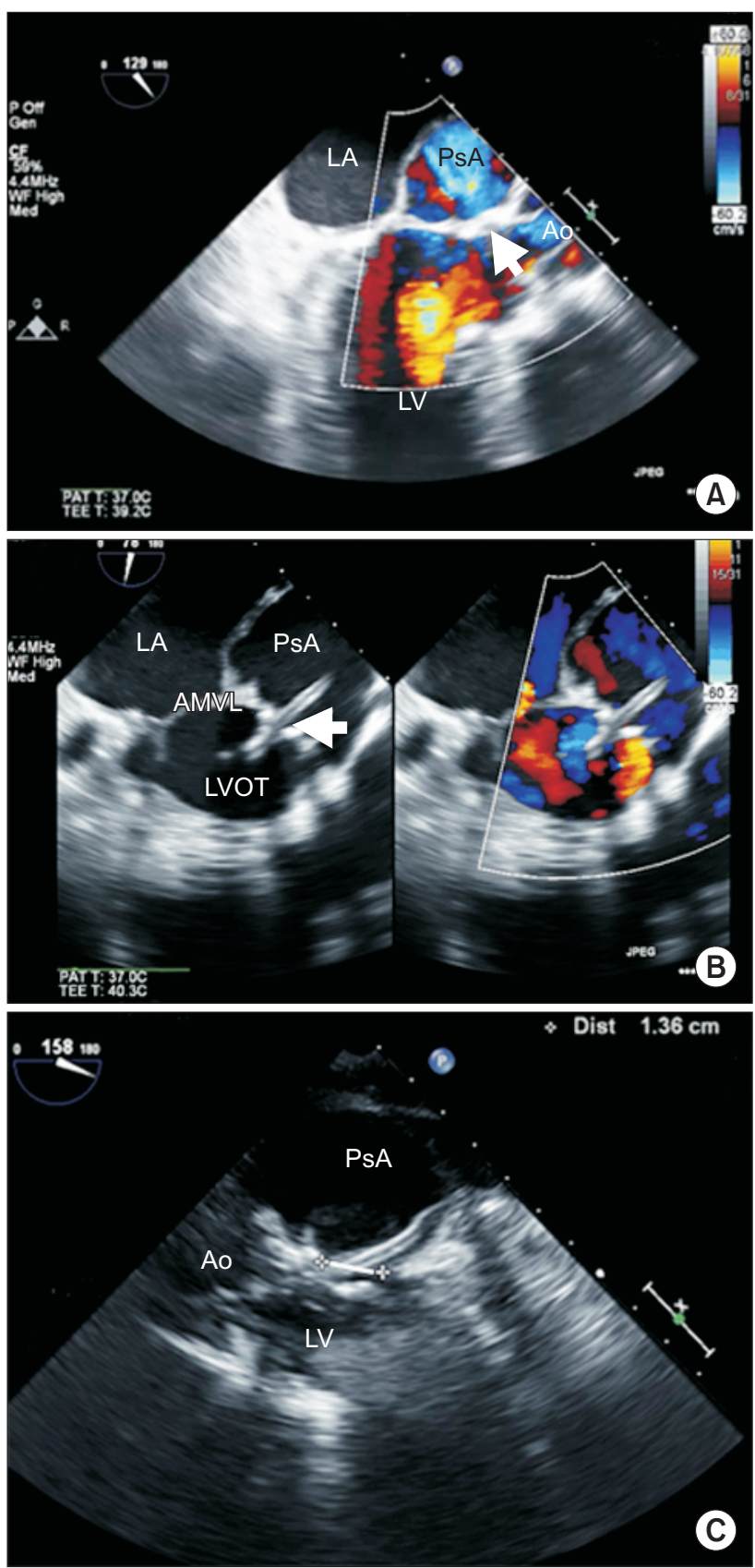

Fig. 2. (A) An intraoperative 2D TEE image with color Doppler, which was taken by slight advancement of the TEE probe from the position of the midesophageal aortic valve short-axis image, delineates the proximal extent of the pseudoaneurysm (PsA) and its communication with the LVOT (arrow). (B) 2D images with color Doppler focused on the neck of the PsA confirm successful advancement of an 8 Fr guiding sheath of the occluding device (arrow) from the ascending aorta into the PsA cavity. (C) The diameter of the aneurysm neck was $13.6 \mathrm{~mm}$ (solid line between cross marks). LA: left atrium, LV: left ventricle, PsA: pseudoaneurysm, Ao: aorta, AMVL: anterior mitral valve leaflet. 


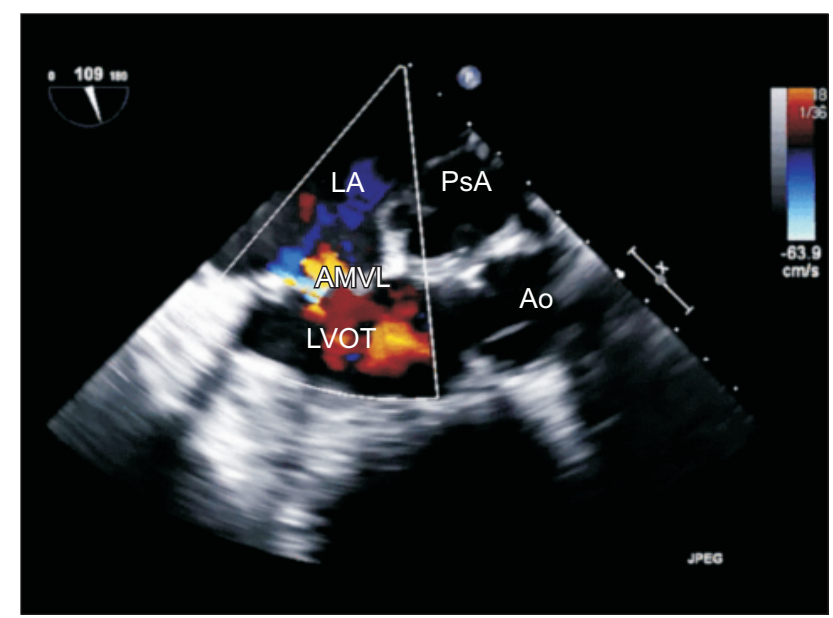

Video 1. Intraoperative two-dimensional (2D) transesophageal echocardiography (TEE). Midesophageal aortic valve long-axis view with color Doppler showing misdirection of the guidewire to the anterior mitral valve leaflet (AMVL). LA: left atrium, AMVL: anterior mitral valve leaflet, LVOT: left ventricle outflow tract, Ao: aorta, PsA: pseudoaneurysm.

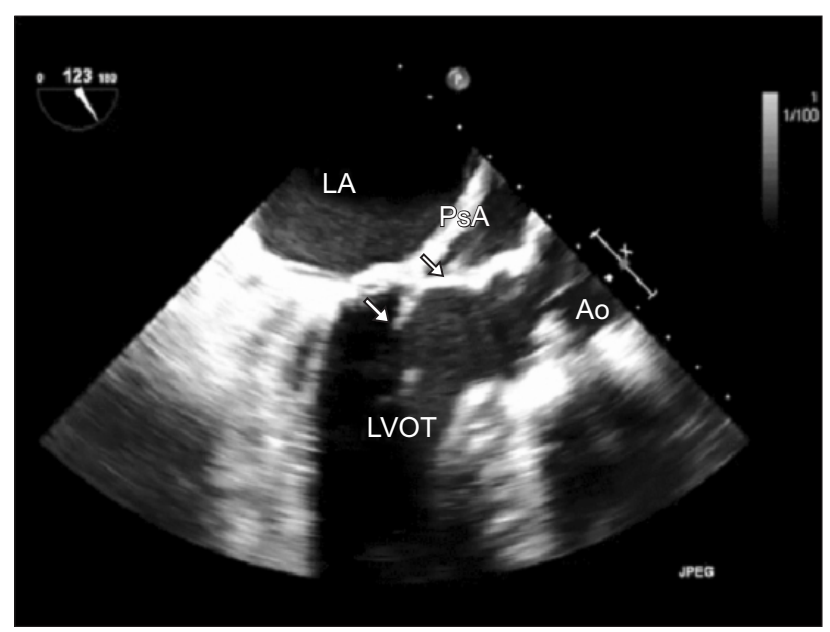

Video 2. Intraoperative two-dimensional (2D) transesophageal echocardiography (TEE). Midesophageal aortic valve long-axis view showing advancement of the guidewire (arrows) into the PsA lumen. LA: left atrium, LVOT: left ventricle outflow tract, Ao: aorta, PsA: pseudoaneurysm.

mild aortic and mitral regurgitation and the location of PsA, adjacent to the aorto-mitral junction and it was communicating with the LVOT (Fig. 2A). There was no significant pathology related to the previously replaced prosthetic aortic valve.

Before the procedure, 5,000 units of unfractionated heparin were injected intravenously and the activated clotting time measured following the injection was 216 seconds. Using the approach via the right common femoral artery, an 8-Fr guiding sheath was advanced into the LVOT and placed across the neck of the PsA under biplane fluoroscopy and TEE guidance (Fig. 2B). Fluoros-
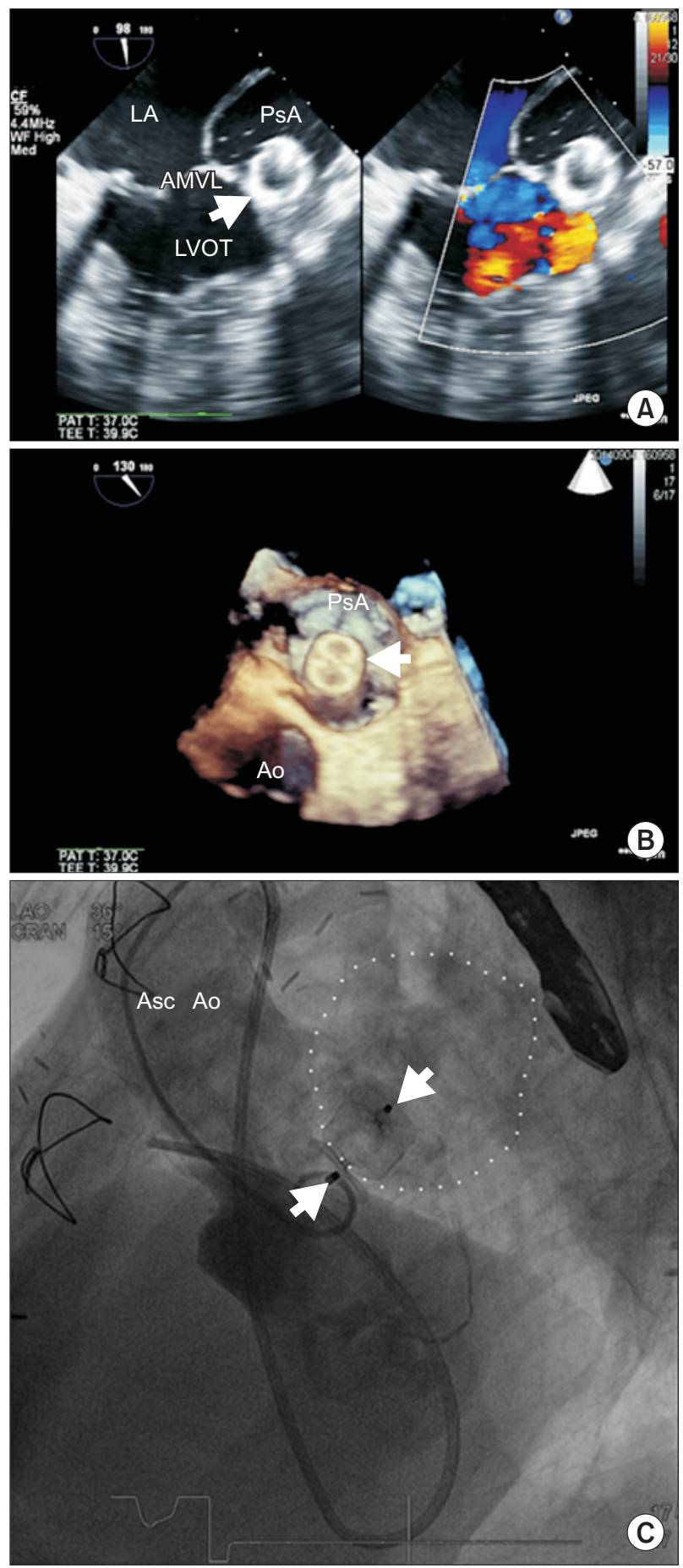

Fig. 3. (A) A 2D image with color Doppler, which was taken immediately after deploying the vascular plug device (arrow), shows diminished Doppler flow pattern within the PsA lumen. (B) 3D enface TEE image of the deployed disc (arrow) seen from the perspective of the PsA cavity. (C) Confirmatory angiography shows diminished flow within the PsA lumen (dotted line). One piece of the type II Amplatzer vascular plug (arrows) is composed of 3 discs and a waist pin that passes through the center. Two out of the 3 discs are located across the PsA lumen and the remaining 1 disc is located outside the PsA lumen. LA: left atrium, PsA: pseudoaneurysm, AMVL: anterior mitral valve leaflet, LVOT: left ventricular outflow tract, Ao: aorta, Asc Ao: ascending aorta. 


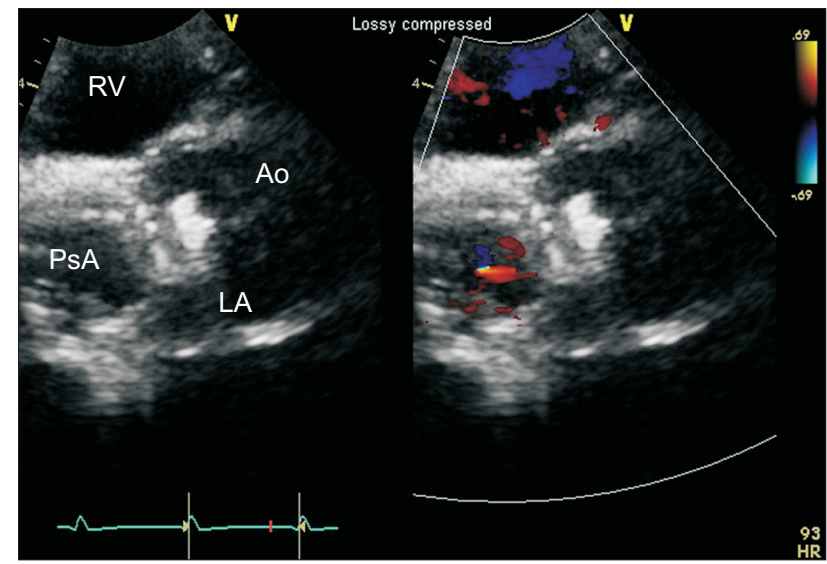

Fig. 4. Postprocedural TTE image with color Doppler on the following day after the procedure. The color Doppler pattern shows diminished flow within the PsA lumen. RV: right ventricle, Ao: aorta, LA: left atrium, PsA: pseudoaneurysm, arrows: vascular plug device.

copy showed a pulsating pouch derived from the aorta. Simultaneously, TEE demonstrated a wide jet of swirling arterial blood flow within the PsA. The diameter and the length of aneurysm neck measured by intra-procedural TEE were $13.6 \mathrm{~mm}$ and 8.6 $\mathrm{mm}$, respectively (Fig. 2C). During the first several attempts to advance the guidewire into the lumen of the PsA, the guidewire passed through the mitral leaflet into the left ventricle, which caused mitral regurgitation (Video 1). After each attempt, we discussed and modified the direction of the guidewire. Finally, the guidewire was successfully advanced into the lumen of the PsA after several failed attempts (Video 2).

A 16-mm-diameter type II Amplatzer vascular plug (AVP, AGA Medical Corporation, MN, USA) was chosen and positioned across the neck of the PsA. After confirming the acceptable position and the intact aortic and mitral valvular function using angiography and TEE, the AVP was deployed. Successful deployment without leakage flow around the AVP was confirmed through the following TEE examination (Figs. 3A and 3B). Angiography also showed diminished flow communication in the PsA (Fig. 3C). After the uneventful procedure, the patient was transferred to the post-anesthesia care unit. The post-procedural TTE also showed no residual flow in the communicating pouch on the aortic root PsA and adequate position of the device (Fig. 4). The post-procedural course was unremarkable and the patient was discharged on post-procedural day 3 without any complications.

\section{Discussion}

Aortic PsA is a rare postoperative complication of cardiac surgery, probably due to graft infection, aortic dissection, connective tissue disorders, and excessive use of biologic glue [1-3].
It usually arises from a dehiscence in the mitral-aortic intervalvular fibrosa [8] and the most common site is previous aortic suture line after cardiac surgery. Development of the LVOT PsA, as in our case report, is very rare. We considered the patient's history of undergoing multiple surgeries of the mitral valve and aorta, as the predisposing factor.

Half of the PsAs are asymptomatic and detected by chance on the routine follow-up imaging study [2-4]. Although the PsA is asymptomatic, its clinical course can be unpredictable and rupture of PsA can be lethal. Cardiac PsAs are reported to have a $30-45 \%$ risk of rupture if left untreated [9]. Therefore, surgical repair has been generally considered as the treatment of choice regardless of the presence of symptoms; however, sometimes it can be challenging with reported mortality rates ranging from $6.7 \%$ to $29 \%[3,4,9]$. In the last decade, percutaneous endovascular closure of PsA was developed as a less invasive alternative treatment for high surgical risk patients $[7,10]$. The treatment option of endovascular approach has been reported including intra-arterial thrombin injection, coil embolization, aortic stent graft insertion and closure using an Amplatzer septal occluder device.

The risk of redo surgery was expected to be prohibitively high because of severe kyphoscoliosis and history of repeated cardiac surgery. Therefore, we selected the endovascular approach rather than surgical repair. Among the options of endovascular approach, we chose the closure using Amplatzer vascular plug. The PsA was located adjacent to the aorto-mitral junction and the left coronary ostium. Stent graft insertion and coil embolization were ruled out due to the inadequate length of landing zone. Intra-arterial thrombin injection was excluded because of the relatively large size of the PsA $(33 \mathrm{~mm})$ [11].

When using the septal occluder device, careful case selection is needed due to the concern related to its mechanical effects on the coronary arteries or valves [11,12].

First, the neck diameter of the aneurysm was between 11 and $13 \mathrm{~mm}$ based on preoperative CT angiography 2 months before the procedure. The 14-mm-diameter type II AVP was initially planned to be used. However, the diameter was measured as $13.6 \mathrm{~mm}$ by intra-procedural TEE, and therefore, the $16-\mathrm{mm}$ diameter type II AVP was finally selected.

Second, in our case, the advancement of guidewire through the PsA opening was expected to be difficult because of the steep angulation between the PsA and LVOT. Simultaneous TEE monitoring enabled advancement of the guidewire into the PsA lumen through several attempts. This also contributed to reducing the total dose of radiocontrast dye, which is potentially nephrotoxic.

Lastly, intra-procedural TEE monitoring allowed our intervention team to check continuously and intermittently for any ventricular systolic or valvular dysfunctions related to the de- 
ployment of the AVP. There was a concern about potential valvular dysfunction after deployment of the AVP due to the very short distance from the aortic valve to the opening of the PsA. Therefore, prior to deploying the AVP, aortic and mitral valvular function as well as any coronary disruption should be evaluated by using both TEE and fluoroscopy. After positioning of the AVP, we also checked the regional wall motion. Procedural complications could be effectively detected and handled immediately under concurrent TEE monitoring rather than single fluoroscopy guidance only [7]. In addition, neither elevation nor depression of ST segments was observed during the procedure in leads II and V. One piece of AVP is composed of 3 discs and a waist pin that passes through the center. We deployed 2 out of the 3 discs across the PsA lumen because positioning the 2 discs within the PsA lumen has been suggested to improve device stability and to alleviate the risk of embolization [10].

Recently, concurrrent TEE monitoring has routinely been used during the procedure using AVPs in many confidential centers $[11,13,14]$. Successful percutaneous closure of a large ascending aortic PsA using an Amplatzer occluder device under TTE guidance has been reported. TTE could be a less invasive alternative to TEE during the procedure, but it compromises real-time fluoroscopic guidance. However, if the PsA opening is located high (probably in the distal ascending aorta), the procedure without TEE guidance could be challenging [14].

The number of anesthetic interventions for diagnostic and interventional procedures performed outside of the operating room as well as the types of the procedures over the past few decades has been growing [15]. Because of the complexity of the procedures and the patients' conditions, there is a wide variety in the anesthetic management options in this area. Therefore, proficient handling of all kinds of monitoring devices and precise analysis of data from these devices are also becoming more and more important in the anesthesia outside of the operating room. Moreover, considering the recent reports of successful anesthetic management using TEE in this field $[6,7,11,13,14]$, efficient use of TEE would be one of the essential requirements for successful anesthesia.

In conclusion, the percutaneous endovascular approach would be an alternative treatment option for the management of aortic PsA in high surgical risk paients. During this intervention, concurrent TEE monitoring with conventional fluoroscopy could provide critical assistance in determining the precise size of the PsA, guiding the catheter, deciding adequate device selection, and identifying complications. Therefore, it would be reasonable to use both TEE and fluoroscopy for successful percutaneous endovascular intervention in patients with aortic PsA.

\section{ORCID}

Hyo Jin Kim, http://orcid.org/0000-0002-7899-3925

Jong-Hwan Lee, http://orcid.org/0000-0001-8249-5550

\section{References}

1. Katsumata T, Moorjani N, Vaccari G, Westaby S. Mediastinal false aneurysm after thoracic aortic surgery. Ann Thorac Surg 2000; 70: 54752.

2. Malvindi PG, van Putte BP, Heijmen RH, Schepens MA, Morshuis WJ. Reoperations for aortic false aneurysms after cardiac surgery. Ann Thorac Surg 2010; 90: 1437-43.

3. Atik FA, Navia JL, Svensson LG, Vega PR, Feng J, Brizzio ME, et al. Surgical treatment of pseudoaneurysm of the thoracic aorta. J Thorac Cardiovasc Surg 2006; 132: 379-85.

4. Malvindi PG, Cappai A, Raffa GM, Barbone A, Basciu A, Citterio E, et al. Analysis of postsurgical aortic false aneurysm in 27 patients. Tex Heart Inst J 2013; 40: 274-80.

5. Jha AK, Pandey R, Gharde P, Devagourou V, Kiran U. Idiopathic left ventricular outflow tract pseudoaneurysm. Ann Card Anaesth 2013; 16: 209-11.

6. Al-Maskari S, Panduranga P, Al-Farqani A, Thomas E, Velliath J. Percutaneous closure of complex paravalvular aortic root pseudoaneurysm and aorta-cavitary fistulas. Indian Heart J 2014; 66: 358-62.

7. Yin WH, Wei J, Tsai SK, Hsiung MC, Lee YT, Yu HP, Ou CH. Transcatheter intervention for complex ascending aortic pseudoaneurysm after cardiac surgery. Circ J 2014; 78: 2215-8.

8. Da Col U, Ramoni E, Di Bella I, Ragni T. An unusual left ventricular outflow pseudoaneurysm: usefulness of echocardiography and multidetector computed tomography for surgical repair. Cardiovasc Intervent Radiol 2009; 32: 188-91.

9. Sullivan KL, Steiner RM, Smullens SN, Griska L, Meister SG. Pseudoaneurysm of the ascending aorta following cardiac surgery. Chest 1988; 93: $138-43$.

10. De Boo DW, Mott N, Kavnoudias H, Walton A, Lyon SM. Endovascular closure of ascending aortic pseudoaneurysm with a type II Amplatzer vascular plug. Vasc Endovascular Surg 2014; 48: 329-32.

11. Kumar PV, Alli O, Bjarnason H, Hagler DJ, Sundt TM, Rihal CS. Percutaneous therapeutic approaches to closure of cardiac pseudoaneurysms. Catheter Cardiovasc Interv 2012; 80: 687-99. 
12. Vola M, Gerbay A, Campisi S, Duprey A, Heller F, Patoir A, et al. Endovascular repair of mitroaortic intervalvular fibrosa aneurysm after bentall surgery. Ann Thorac Surg 2015; 99: 702-4.

13. Noble S, Ibrahim R. Embolization of an Amplatzer mVSD occluder device used for percutaneous closure of an ascending aortic pseudoaneurysm: case report and literature review. Catheter Cardiovasc Interv 2012; 79: 334-8.

14. Vavuranakis M, Kalogeras K, Moldovan C, Vaina S, Vrachatis D, Kariori M, et al. Percutaneous closure of a large ascending aorta pseudoaneurysm due to mediastinitis using an amplatzer occluder device. JACC Cardiovasc Interv 2015; 8: 495-7.

15. Metzner J, Domino KB. Risks of anesthesia or sedation outside the operating room: the role of the anesthesia care provider. Curr Opin Anaesthesiol 2010; 23: 523-31. 